\title{
Analysis of long-term single-site observations of the pulsating DB white dwarf GD $358^{1}$
}

\author{
P.A. Bradley \\ Los Alamos National Laboratory, X-2 MS B220, Los Alamos, NM \\ $87545-2345, U S A$
}

\begin{abstract}
We briefly describe the analysis of single-site data collected from 1982 to 1992 for the pulsating DB white dwarf GD 358. We show that the amplitudes of almost all of the $\ell=1$ modes change over time, and some of the $\ell=1$ modes disappear for months at a time. We also find a new mode at $k=19$, with a frequency of $\sim 1173 \mu \mathrm{Hz}$.
\end{abstract}

\section{Introduction}

The pulsating DB white dwarf GD 358 was observed by the Whole Earth Telescope (WET) in 1990, 1994, and 2000. While these observing runs revealed a wealth of pulsation modes, they constitute only three "snapshots" of the behavior of this star. These "snapshots" show that GD 358 has a series of $\ell=1$ modes present in the period range of 420 to $810 \mathrm{~s}$, with numerous Fourier Transform peaks at the sums and differences of the $\ell=1$ mode frequencies. In addition, the amplitudes of the $\ell=1$ modes and the sum and difference frequency peaks (which we also call "combination peaks" in this paper) are different in each WET run. These data are not sufficient to determine the time scale of the amplitude changes and whether additional $\ell=1$ modes might be present. For this, we need more frequent data sets, although not necessarily WET data.

To this end, we and others collected single-site data on this star from 1990 to 1992 and we have analyzed archival data from 1982 to 1986 . We use the WET data to determine the periods of the pulsation modes and take this information to disentangle the pulsation modes from the extensive alias pattern. We have data from 1985 April, 1986 May, 1990 June and August, 1991 April and May, and 1992 early and late June.

\section{Data reduction methodology}

Nather et al. (1990), Kepler (1993), and Clemens (1993) describe the general data reduction process in detail, so we will only mention the highlights here. First, we use a data reduction program called QED to eliminate bad data points. We then use QED to perform dead-time corrections, sky subtraction, extinction correction, and convert from counts to fractional intensity (with the mean value

\footnotetext{
${ }^{1}$ These data were collected at McDonald Observatory, Fort Davis, Texas
} 
of the data being used as the zero point). In some cases, the extinction correction left long-term trends in individual runs, and we subtract low-order polynomials to flatten out the individual light curves. Once we obtain the reduced data, we compute Fourier Transforms (FT) for the combined runs of a given month, along with a representative window function. For this preliminary analysis, we compare the frequencies from each month to those of the WET runs to determine their reality, and we use the window function to reject alias peaks. For larger amplitude modes, we can also ascertain the reality of a mode by looking for combination peaks (peaks at the sum and/or difference frequency of two modes).

\section{Results}

Winget et al. (1994), Vuille et al. (2000), and Kepler et al. (2003) have FT plots for GD 358 that look somewhat similar to each other. In Fig. 1, we show an FT plot from 1986 May that looks rather different to the previously published plots. In the main $\ell=1$ region from 1000 to $2000 \mu \mathrm{Hz}$ (see Fig. 1), we see the $k=19,18,15,14$, and 13 modes. The $k=9$ and 8 modes are also present. Most FT plots show the $k=17$ mode at high amplitude, and it is not present in 1986 May. While we have seen all of these $\ell=1$ modes before, we have not normally seen the $k=13$ and 14 modes at such large amplitudes. As a result, these combination peaks hinder our ability to see modes with periods longer than $810 \mathrm{~s}$ or shorter than $420 \mathrm{~s}$. However, we see evidence of a mode at $k=19$ in the 1985 April and 1986 May data sets that was also seen by Kepler et al. (2003). The period of this mode is about $854 \mathrm{~s}$, and the period spacing is $44 \mathrm{~s}$, implying that the next trapped mode must be at $k=20$ or greater, since we have not found a period spacing minimum yet.

There is also rich spectrum of combination peaks, of which the most prominent ones are labelled in Fig. 1. Combination peaks result from a distortion of the parent mode eigenfuctions as they move through the surface convection zone of the star (Wu 2001; Brickhill 1992). We can easily identify up to third order sum frequency peaks, such as $19+14+14$ at $4209.9 \mu \mathrm{Hz}$. There may be even higher order peaks, but we have not yet searched for them. There are also some difference combination peaks, but not nearly as many as sum peaks. We also note that while the sum combination peaks originate from the largest amplitude modes, there is not an obvious correlation of combination peak amplitude with parent mode amplitude. One example of this anticorrelation is that the amplitude of the $19+15$ mode is larger than the $18+14$ mode. Some of the combination peaks defy an easy explanation. A good example is the prominent peak at $4037.8 \mu \mathrm{Hz}$.

The data show that all of the major pulsation modes (which lie between 400 and 850 s) vary in amplitude (see Table 1), and some modes even disappear below the noise limit for months at a time. The lowest overtone modes at 424 and $464 \mathrm{~s}(k=8$ and 9$)$ are present in all the data, and they vary in amplitude by up to a factor of 4 . We find no convincing evidence of modes at $\sim 500$ or $\sim 540 \mathrm{~s}(k=10$ and 11), even though the models of Bradley \& Winget (1994) predict modes near these periods. The $k=13$ through 19 region almost always dominates the power spectrum and the amplitudes of these modes can grow to $35 \mathrm{mma}$ (milli-modulation amplitude), although values of 20 to $25 \mathrm{mma}$ are 


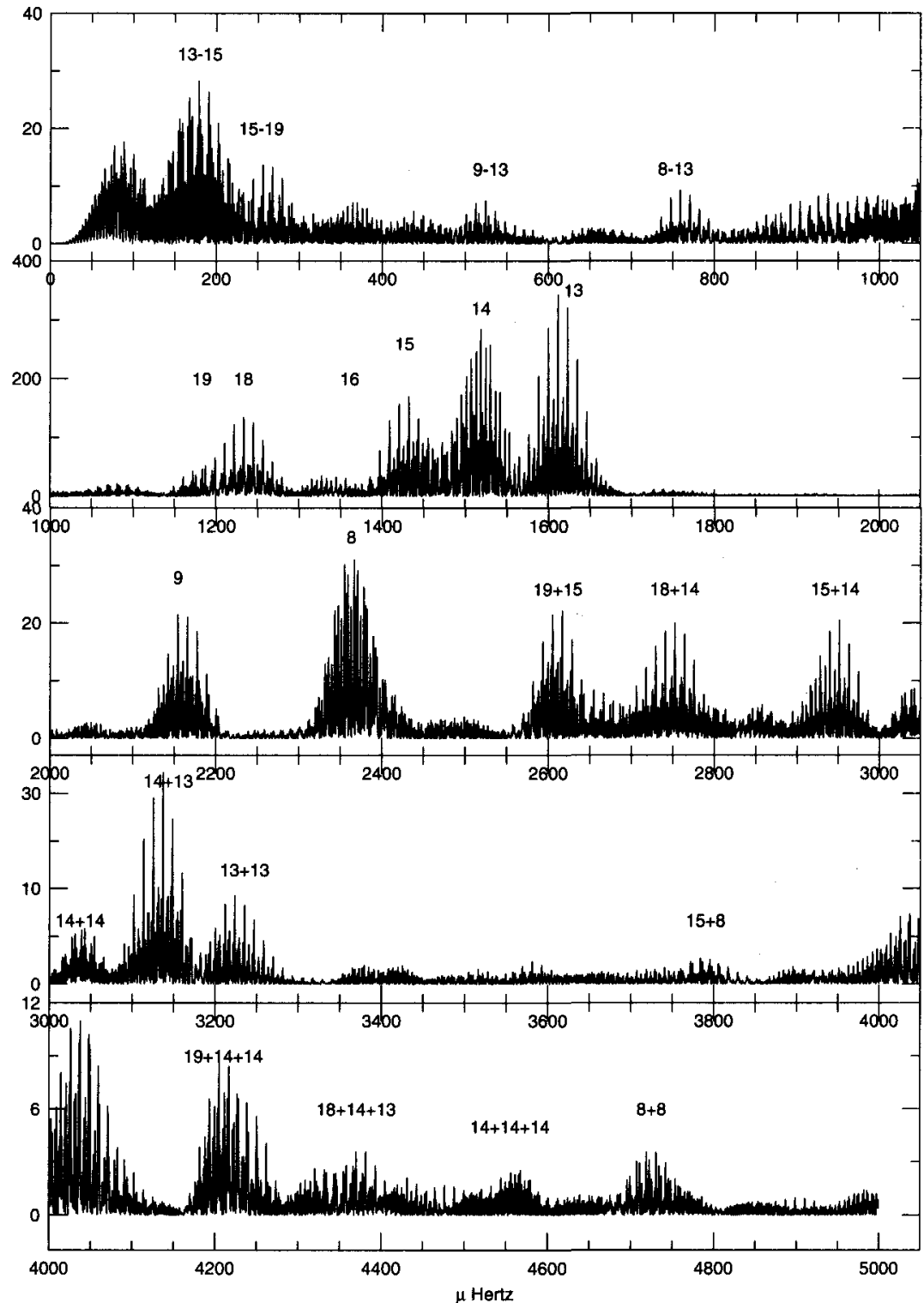

Figure 1. A Fourier power Transform from 1986 May. The vertical units are amplitude squared (power) and the frequency is in $\mu \mathrm{Hz}$. The numbers above the prominent peaks are the $\ell=1$ overtone numbers of the modes. Peaks with two numbers, such as $18+14$ are sum combination peaks. 
typical. The frequencies of these modes can change by 1 to $2 \mu \mathrm{Hz}$ on timescales of a few months or less.

Table 1. Main periodicities in 1985 April, 1986 May, 1990 May, and 1991 May.

\begin{tabular}{lcrcrcrrr}
\hline & \multicolumn{2}{c}{ April 1985 } & \multicolumn{2}{c}{ May 1986 } & \multicolumn{2}{c}{ May 1990} & \multicolumn{2}{c}{ May 1991 } \\
\hline $\mathrm{k}$ & $\begin{array}{c}\text { Freq. } \\
(\mu \mathrm{Hz})\end{array}$ & $\begin{array}{r}\text { Amp. } \\
(\mathrm{mma})\end{array}$ & $\begin{array}{c}\text { Freq. } \\
(\mu \mathrm{Hz})\end{array}$ & $\begin{array}{r}\text { Amp. } \\
(\mathrm{mma})\end{array}$ & $\begin{array}{c}\text { Freq. } \\
(\mu \mathrm{Hz})\end{array}$ & $\begin{array}{r}\text { Amp. } \\
(\mathrm{mma})\end{array}$ & $\begin{array}{c}\text { Freq. } \\
(\mu \mathrm{Hz})\end{array}$ & $\begin{array}{r}\text { Amp. } \\
(\mathrm{mma})\end{array}$ \\
19 & 1170.49 & 26.48 & 1172.33 & 6.66 & & & 1173.64 & 4.54 \\
18 & 1232.86 & 12.92 & 1233.59 & 11.72 & 1233.41 & 5.05 & 1234.12 & 4.13 \\
17 & 1297.60 & 19.61 & 1297.02 & 2.63 & 1297.59 & 14.60 & 1296.72 & 21.68 \\
16 & 1361.14 & 5.00 & 1361.76 & 4.10 & 1361.71 & 2.21 & 1361.76 & 3.25 \\
15 & 1426.97 & 5.05 & 1427.23 & 5.10 & 1427.40 & 19.24 & 1426.78 & 3.84 \\
14 & 1517.54 & 2.33 & 1518.72 & 16.91 & 1518.99 & 9.71 & 1518.27 & 1.62 \\
13 & 1618.34 & 3.31 & 1617.99 & 12.98 & 1617.30 & 5.28 & 1616.90 & 1.99 \\
12 & 1735.12 & 1.01 & 1734.70 & 2.52 & 1733.85 & 0.53 & 1738.06 & 1.18 \\
9 & 2154.49 & 2.42 & 2154.00 & 4.64 & 2154.05 & 4.59 & 2154.24 & 3.84 \\
8 & 2361.82 & 6.29 & 2362.58 & 4.91 & 2362.59 & 5.77 & 2362.54 & 5.46 \\
\hline \hline
\end{tabular}

We show that the preliminary analysis of single-site data from 1982 through 1986 and then from 1990 through 1992 provides a valuable compliment to the WET data sets of 1990,1994 , and 2000 . We see that the main $\ell=1$ modes vary in amplitude from month to month, with some of the modes (such as $k=17$ ) disappearing for several months. We confirm the presence of the $k=19$ mode that Kepler et al. (2003) report and show that it too has a variable amplitude. We also see a rich spectrum of sum and difference combination peaks that changes from run to run as the parent $\ell=1$ modes change in amplitude. Our analysis of this data set will continue, and we hope to have at least the Fourier Transform plots and frequency results ready for a paper before too long. More time will be required to attempt to correlate the behavior of the combination peaks with their parent modes, although this will provide valuable data for nonlinear pulsation theory.

\section{References}

Bradley, P.A., Winget, D.E. 1994, ApJ, 430, 850

Brickhill, J.A. 1992, MNRAS, 259, 529

Clemens, J.C. 1993, Baltic Astron., 2, 511

Kepler, S.O. 1993, Baltic Astron., 2, 515

Kepler, S.O. et al. 2003, A\&A, 401, 639

Nather, R.E., Winget, D.E., Clemens, J.C., Hansen, C.J., Hine, B.P. 1990, ApJ, 361,309

Vuille, F., et al. 2000, MNRAS, 314, 689

Winget, D.E., et al. 1994, ApJ, 430, 839

Wu, Y. 2001, MNRAS, 323, 248 


\section{Discussion}

Handler: Taken alone, the temporal behaviour of GD 358 may appear freaky, but data I have on two other DBVs confirm virtually all your observational findings. Thus there is certainly some common physics involved (that we don't understand yet.

Bradley: I agree that it is not "freaky", since the changing amplitudes and combination peaks are also seen in the large amplitude DA pulsators.

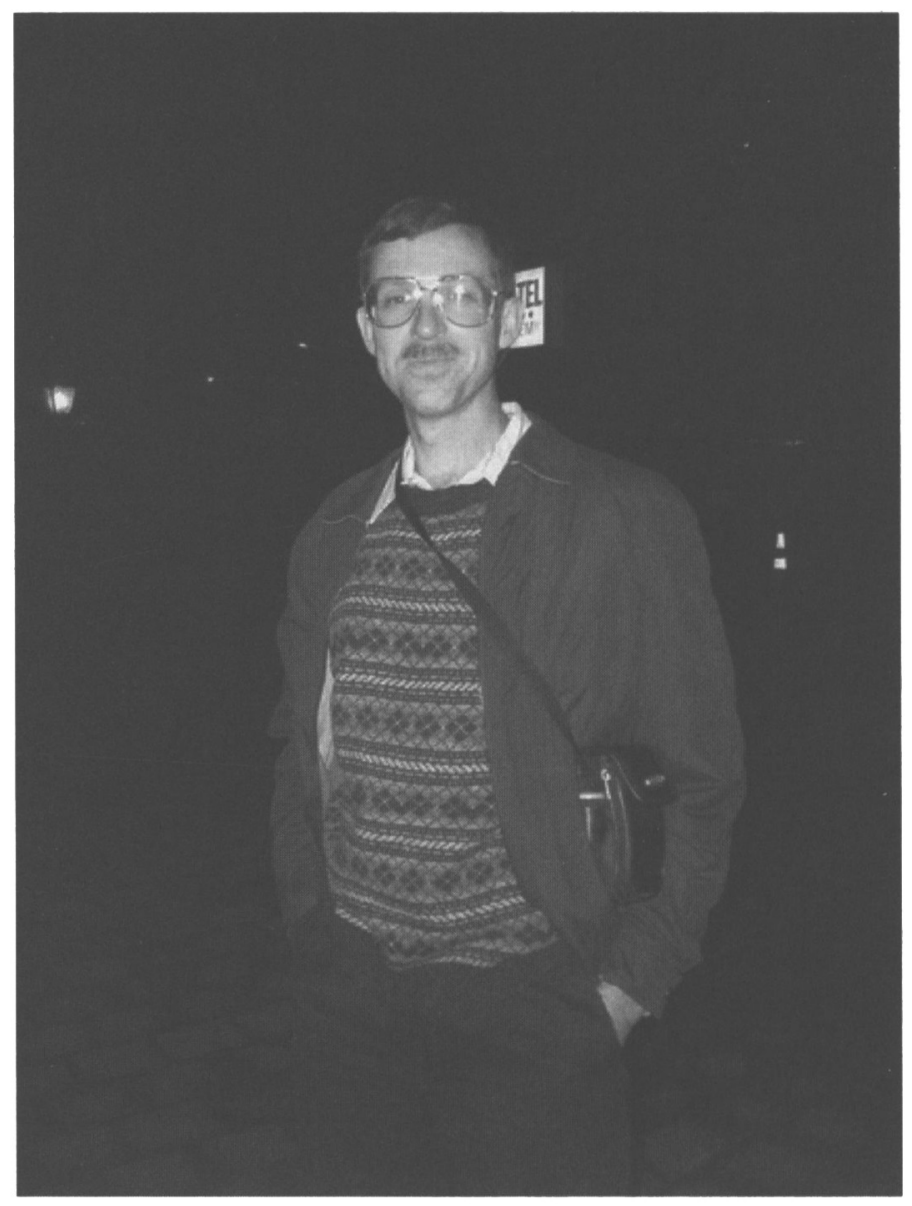

Paul Bradley 